\title{
Review
}

\section{Rationale of screening for early kidney damage in patients with high cardiovascular risk: nephrologist's point of view}

\author{
Laurynas Rimševičius a,b, ${ }^{a}$, Diana Aksionova ${ }^{a, b}$, Marius Miglinas ${ }^{a, b}$, \\ Jolita Badarienè ${ }^{c}$, Ligita Ryliškytė ${ }^{c}$, Alma Čypienè ${ }^{c}$, Vytautas Kasiulevičius a, \\ Mantė Barzdenytè ${ }^{a}$, Justina Tracevičiūtè ${ }^{a}$, Aleksandras Laucevičius ${ }^{a, c}$ \\ a Faculty of Medicine, Vilnius University, Vilnius, Lithuania \\ ${ }^{\mathrm{b}}$ Centre of Nephrology, Vilnius University Hospital Santariškiu Klinikos, Vilnius, Lithuania \\ ${ }^{c}$ Centre of Cardiology and Angiology, Vilnius University Hospital Santariškiu Klinikos, Vilnius, Lithuania
}

Received 13 September 2012; accepted 14 January 2013

\begin{abstract}
Summary
Increased awareness of chronic kidney disease stimulates an interest towards early detection and prevention. The true prevalence of kidney injury varies from 10 to $40 \%$, mostly depending on the methodology of the study and the population enrolled. A screening strategy targeting the highest risk groups, those with diabetes or hypertension, family history of diabetes, hypertension, or kidney disease, is likely to be most efficient and cost effective. Quantification for albuminuria should be performed using laboratory methods or albumin to creatinine ratio and should be monitored at regular intervals. The most correct equations calculating glomerular filtration rate differ in separate populations, and the most accurate equations in patients with high cardiovascular risk are MDRD and CKD-EPI. Markers of early kidney damage have association with other target organs damage, even in subclinical or preclinical mode. Individuals at stage 4 and 5 chronic kidney disease, with higher levels of proteinuria, proteinuria together with haematuria, rapidly declining glomerular filtration rate, or poorly controlled hypertension should be referred to a nephrologist in order to identify the cause, provide recommendations, slow progression, or treat complications.
\end{abstract}

Seminars in Cardiovascular Medicine 2013; 19:1-8

Keywords: chronic kidney disease, glomerular filtration rate, high cardiovascular risk, microalbuminuria, screening

\section{Introduction}

Chronic kidney disease (CKD) is present in far more people than is anticipated, and patients with this condition have a higher burden load of cardiovascular disease. In developed countries, the increase in prevalence of CKD has been attributed to an increase in stipulating factors, such as hypertension and diabetes. Also, presence of CKD is a well-known forerunner provoking cardiovascular events. The underlying kidney damage permits us to classify patients to the same category of risk for cardiovascular death as those with severe notorious heart disease or diabetes.

\footnotetext{
* Corresponding address: Laurynas Rimševičius, Clinic of Gastroenterology, Nephrourology and Surgery, Faculty of Medicine, Vilnius University, M.K. Čiurlionio str. 21, Vilnius 03101, Lithuania.

E-mail: laurynas.rimsevicius@santa.lt (L. Rimševičius).
}

The declining kidney function may increase this risk in an extent-dependent way.

True data on the prevalence of CKD is still emerging in many countries with shortage of integral and standardized prophylactic care in primary health system, which should focus on early kidney damage. The implemented methods evaluating renal function are highly underutilized, thought mostly are cheap and non-complicated. Despite changing recommendations in this area, improvement in diagnosis of CKD in patients with high cardiovascular risk may lead to generally improved health outcomes, especially considering high costs and poor outcomes in individuals with end stage kidney disease.

This article focuses on main methods that are recommended to screen for kidney damage in individuals with high cardiovascular risk for CKD. We recommend the patterns to estimate glomerular filtration rate and albuminuria, which fit the 
Table 1.

Early kidney damage or CKD in general population

\begin{tabular}{|c|c|c|c|c|c|c|c|c|}
\hline Population & Survey & Renal function & CKD1 & CKD2 & CKD3 & CKD4 & CKD5 & Reference \\
\hline 95,255 & Open screening offer $>20$ years & Cocroft-Gault & - & - & 40.0 & 2.5 & 0.1 & Japan [45] \\
\hline 11,247 & Open screening offer $>20$ years & $\begin{array}{l}\text { Cocroft-Gault, } \\
\text { adopted for BSA }\end{array}$ & 0.9 & 2.0 & 10.9 & 0.3 & 0.003 & Australia [46] \\
\hline 19,256 & Pop. survey $\geq 30$ years & MDRD & - & - & 8.3 & - & - & Iceland [47] \\
\hline 13,251 & $\begin{array}{c}\text { NHANES III } \geq 20 \text { years, } \\
\text { no DM }\end{array}$ & MDRD & - & - & 3.2 & 0.16 & - & USA [48] \\
\hline 15,625 & NHANES III $\geq 20$ years & MDRD & 3.3 & 3.0 & 4.3 & 0.2 & 0.2 & USA [49] \\
\hline 4,101 & NHANES III $\geq 20$ years & MDRD & 2.8 & 2.8 & 3.7 & 0.13 & - & USA [50] \\
\hline 65,181 & Pop. survey $\geq 20$ years & MDRD & 2.7 & 3.2 & 4.2 & 0.16 & - & Norway [51] \\
\hline
\end{tabular}

CKD - chronic kidney disease, NHANES - National Health and Nutrition Examination Survey, MDRD - Modification of Diet in Renal Disease, BSA - body surface area.

patients with high cardiovascular risk. Also, the article describes recently found associations of early kidney damage with subclinical changes in the target organs.

\section{Prevalence of renal impairment in general versus at-risk population}

High prevalence of CKD is determined by noncontagious and also non-intrinsic renal disorders, including hypertension, diabetes, and the aging. Increased awareness of CKD has direct interest toward early detection and prevention. Recognition of CKD has improved since the introduction of glomerular filtration rate (GFR) reporting and CKD guidelines, but in some cases this reveals ascertainment bias rather than a true increase in prevalence [1].

The true data of the prevalence of early kidney damage or CKD in general population and in high-risk individuals varies, mostly depending on the methodology of the study [2]. Data from international studies that were performed in general population are shown in the Table 1 .

High rates of CKD could be conditioned by definition and classification of the disease. As currently defined and detected, screening programs mainly identify individuals with sole microalbuminuria (CKD stage 1 and stage 2) or reduced GFR (CKD stage 3), and in some persons it could lead to a wrong diagnosis, if tests are not repeated or made correctly. A perfect screening strategy should include randomization of individuals to early detection followed by close monitoring for further tests, complications of the disease and the benefit of screening on reducing mortality. Such an experimental design would reduce the risk of selection, overdiagnosis and other biases. The available evidence for screening of CKD is mostly based on observational studies, and no such an ideal trial of screening exists in CKD. Population surveys are usually based on a single blood or urine sample. Also, most of the perceived high prevalence of CKD is accounted for by individuals over the age of 60 years, who have a physiological decline in kidney function with age. In this population the rate of decline in renal function in the absence of comorbidities may be less than previously thought [1], and GFR will not decline to a clinically significant level.

To avoid this inaccuracy, one must perform screening for individuals, who are at risk, but not general population. A screening strategy for CKD targeting the highest risk groups, those with diabetes or hypertension, family history of diabetes, hypertension, or kidney disease, is likely to be most efficient and cost effective. Table 2 shows the at-risk population and additional atrisk groups, that could benefit from the screening for renal impairment, according to main published guidelines.

The National Kidney Foundation's Kidney Early Evaluation Program (KEEP) in United Kingdom has screened more than 100,000 individuals at risk, of whom $28.7 \%$ had CKD, with a prevalence of stage $1-3.1 \%$, stage $2-4.8 \%$, stage $3-19.7 \%$; and stages 4 and $5-1.1 \%$ [6]. This distribution demonstrates sufficient detecting of individuals in the early stages of CKD. Other studies show the CKD progression risk, e.g. in the 12,866 participants of the Multiple Risk Factor Intervention Study (MRFIT), followed for 25 years for end stage renal disease (ESRD) development, dipstick proteinuria $\geq 2+$ and GFR $\leq 60$ were associated with a 41-fold higher risk of ESRD [7]. Also, there is an urgent need for further research to support service change for reducing the progression of 
Table 2.

At-risk groups for CKD in different guidelines

\begin{tabular}{ll}
\hline & The at-risk population \\
\hline Most guidelines & Established kidney disease \\
& Vascular disease \\
& Hypertension \\
& Diabetes \\
& Family history of CKD \\
& Those with symptoms or at high \\
& risk of obstructive uropathy \\
& Additional at-risk groups \\
\hline UK guidelines [3] & Heart failure \\
& Nephrotoxic drugs \\
& Multisystem disease with potential \\
& renal involvement \\
& All health encounters \\
& Multisystem disease with potential \\
KDOQI guidelines [4] & renal involvement \\
& US ethnic minority status \\
& Aboriginal Australians \\
& Torres Strait Islanders
\end{tabular}

CKD and CKD complications, when recognized the number of individuals as having markers of renal function impairment.

\section{Definition and classification of CKD in patients with cardiovascular risk}

To treat individuals with CKD and improving the outcomes, a coordinated overall approach is needed for identifying the earlier stages of disease, also detecting the populations at increased risk. The National Kidney Foundation Disease Outcomes Quality Initiative (K/DOQI) guidelines proposed a classification system for CKD based on the level of GFR and presence of kidney damage (Table 3) and it is widely accepted international societies and groups [8]. Other modifications of this classification are shown in Table 4 , but generally these reflect the original classification.

The definition of CKD compiles the measurement of reduced renal function (GFR) and of renal parenchymal injury such as proteinuria and/or anatomical abnormalities. The presence of kidney damage for at least 3 months is considered to be the sufficient index for diagnosing CKD. Also, the persisting decreased function or kidney damage for at least three months is necessary to distinguish CKD from acute kidney injury.

Many individuals should be referred to a nephrologist in order to identify the cause of the CKD and to provide recommendations for therapies to reverse the kidney disease, slow progression, or treat complications. According to most guidelines, individuals at stage 4 and $5 \mathrm{CKD}$, with higher levels of proteinuria unless known to be due to diabetes, already appropriately treated proteinuria together with haematuria and rapidly declining GFR, poorly controlled hypertension, suspected of having genetic causes of CKD or suspected renal artery stenosis should be evaluated by a nephrologist.

\section{Estimation of glomerular filtration rate: using the right equation}

The GFR is considered to be the best indicator of renal function. Usually it is described as the volume of fluid that is filtered from the glomerular capillaries per time unit. The gold standard for GFR assessment is the renal inulin clearance, likewise nonradioactive contrast agents or radiolabeled isotopes [10]. Also, a traditional method for estimating GFR is calculation of the creatinine clearance from serum creatinine and a 24-hour urine collection. This method is unreliable, when the urine collection is being performed at home. These tests cannot be used routinely in daily clinical practice, and few creatinine-based formulas were created for predicting GFR, as serum creatinine is the regularly used measurement to evaluate renal function. Serum creatinine alone should not be used to assess the level of kidney function because of inter-individual variation in creatinine production, tubular and extra-renal secretion of creatinine, and the laboratory differences between analytical methods [11].

Table 3.

Staging of chronic kidney disease [4]

\begin{tabular}{|c|c|c|c|c|}
\hline \multirow[t]{2}{*}{ GFR $\left(\mathrm{ml} / \mathrm{min} / 1.73 \mathrm{~m}^{2}\right)$} & \multicolumn{2}{|c|}{ With kidney damage ${ }^{*}$} & \multicolumn{2}{|c|}{ Without kidney damage } \\
\hline & With $\mathrm{HBP}^{* *}$ & Without HBP & With HBP & Without HBP \\
\hline$\geq 90$ & 1 & 1 & HBP & \\
\hline $60-89$ & 2 & 2 & HBP with $\downarrow$ GFR & $\downarrow$ GFR \\
\hline $30-59$ & 3 & 3 & 3 & 3 \\
\hline $15-29$ & 4 & 4 & 4 & 4 \\
\hline$<15$ or dialysis & 5 & 5 & 5 & 5 \\
\hline
\end{tabular}

* Abnormalities in blood, urine tests, imaging studies, ${ }^{* *} \mathrm{HBP}-$ high blood pressure $\geq 140 / 90 \mathrm{mmHg}$ in adults. 
Table 4.

$\mathrm{K} / \mathrm{DOQI}$ classification and updates [9]

\begin{tabular}{|c|c|c|c|c|c|c|}
\hline \multicolumn{2}{|c|}{ K/DOQI classification } & \multicolumn{5}{|c|}{ Other classifications } \\
\hline Stage & $\operatorname{GFR}\left(\mathrm{ml} / \mathrm{min} / 1.73 \mathrm{~m}^{2}\right)$ & KDIGO & & CARI & NICE & \\
\hline 1 & $\geq 90$ & $\mathrm{~T}$, if transplanted & & $\mathrm{P}$, if proteinuria & $\mathrm{P}$, if proteinuria & \\
\hline 2 & $60-89$ & & & & & \\
\hline \multirow[t]{2}{*}{3} & $30-59$ & & & & $\begin{array}{l}\text { Identify rate of } \\
\text { progression }\end{array}$ & 3a (GFR 45-59) \\
\hline & & & & & & 3b (GFR 30-44) \\
\hline 4 & $15-29$ & & & & & \\
\hline 5 & $<15$ or dialysis & & $\begin{array}{l}\mathrm{D} \text {, if on } \\
\text { dialysis }\end{array}$ & & & \\
\hline
\end{tabular}

CARI - Caring. For Australians with Renal Impairment, KDIGO - Kidney Disease: Improving Global Outcomes, K/DOQI National Kidney Foundation's Kidney Disease Outcomes Quality Initiative; NICE - National Health Service - National Institute for Health and Clinical Excellence.

The first equation calculating GFR was published in 1976 by Cockcroft and Gault. It was developed in 249 hospitalized white men with mean age of 57 years and mean estimated creatinine clearance of $73 \mathrm{ml} / \mathrm{min}$ [12]. Historically this equation has been widely used in clinical practice. Nevertheless, this equation is not adjusted for body surface area and is based on predicting the daily creatinine excretion given the age, weight and sex of the patient. Currently, this equation is mostly recommended for dosing medications $[13,14]$.

Another formula, the Modification of Diet in Renal Disease (MDRD) equation, was developed in 1999 from a sample of 1,628 MDRD Study participants. All individuals had CKD, and no transplant recipients or individuals with diabetic kidney disease were included [15]. The equation accounts for creatinine generation by adjusting for race, age and gender, and estimates GFR adjusted for body surface area. It was validated in pooled populations with diverse clinical characteristics, and has gained widespread acceptance. The MDRD formula is the recommended serumcreatinine-based equation by NKF KDOQI $[4,8]$. The most critical limitation of using this equation is that its performance is not accurate for higher levels of GFR and overestimates the prevalence of CKD stage 3 in the general population [16,17].

The MDRD equation was recently updated in 2009 by a new equation developed by the Chronic Kidney Disease Epidemiology Collaboration (CKD-EPI). It was developed and validated using data from 26 studies in which GFR was measured [18]. The population included a large number of older individuals and African Americans. The equation has been shown to be as accurate as that of the MDRD equation for individuals with GFR $<60 \mathrm{ml} / \mathrm{min} / 1.73 \mathrm{~m}^{2}$. The CKD-EPI equation estimates higher GFR values than does the MDRD equation for younger or female individuals, who often have lower risk of complications, but the same serum creatinine level. Also, the CKD-EPI equation classifies fewer individuals as having CKD and more accurately categorizes the risk for mortality and ESRD than did the MDRD Study [19].

The CKD-EPI equation appears to be less biased and was more accurate than the MDRD equation in elderly patients [20], and provides a better definition of cardiovascular disease burden associated with CKD estimating GFR in patients with type 2 diabetes [21]. Overall, the most correct equation should be developed and validated in separate populations. Accuracy is probably the best single measure for comparing equations because it incorporates bias and precision.

\section{Diagnostic methods for albuminuria}

Measurement of urine albumin is an important issue, as albuminuria is a valuable predictor of cardiovascular events. Although, studies provide different clinical recommendations for urine albumin measurement, it still lacks standardization [22]. Due to lack of universal agreement, there are many differences in the urine collecting methods, laboratory measurement procedures and the interpretation of results [23].

The chemistry of albumin in urine is not clearly defined. Various modified albumin forms may be filtered at the glomerulus on account of size or charge differences. Also, receptor-mediated reabsorbtion at the tubules might be influenced by variant molecular forms of albumin. Molecular forms in serum and urine are slightly different after the affect of proteases in the urinary tract. Additionally, there is some evidence of albumin absorption on plastic surfaces [24].

The gold standard for detection of small quantities of urinary albumin is the 24-hour urine 
collection, however it has been suggested that screening can be achieved merely by a time urine collection or early morning single-void urine samples. Moreover, the ratio of the albumin concentration to the creatinine concentration (ACR) is widely viewed as an acceptable substitute for the albumin excretion rate.

Standard urinary dipstick shows colorimetric reaction between albumin and tetrabromphenol blue, producing different shades of green if a certain amount of albumin is present. Therefore, it is not very accurate to evaluate the severity of albumin excretion, because, generally, it becomes positive, if protein excretion reaches $300-500 \mathrm{mg} / \mathrm{d}$. In addition, false negative and false positive results can occur due to the urine albumin concentration as it depends on the urine volume as well as the amount of albuminuria. Also, semiquantitative dipsticks can be used to measure albumin concentration, though sensitivity and specificity of these tests range from 33 to $80 \%$ and from 80 to $97 \%$ respectively [25].

Evaluation of total protein to creatinine ratio (TPCR) or ACR on morning single-void urine samples correlates well with 24-hour total protein and albumin excretion. Preferably ACR to TPCR is used to quantify small amounts of albumin excretion. The diagnostic performance of both tests differs significantly with age, sex and ethnicity. This should be taken into consideration when interpreting results [26]. In addition, ACR should be reported with all the urine albumin measurements.

Variety of laboratory methods is used to measure the albumin excretion directly. Most common approaches are immunology based methods, such as immunonephelometry, immunoturbidimetry or radioimmunoassay. Considering that urine albumin is heterogeneous and has at least five different antigenic sites, there is a discrepancy between reported values depending on the method used [27]. In comparison, high performance liquid chromatography (HPLC) based measurements are able to assess 'nonimmunogenic' albumin, resulting in higher values for the albumin excretion. However, the recent evidence supports the view that the HPLC assays do not resolve albumin from other co-eluting urine proteins and cause overestimation [28]. Further evidence indicates that HPLC method has no advantages over immunoassay as a predictive marker for adverse cardiovascular events [29].

Furthermore, in many laboratories, albumin is measured by automated technique of two dye-binding assays: bromcresol green (BCG) and bromcresol purple (BCP). BCG proves to be less specific as it binds to non-albumin proteins in comparison with BCP. The difference is elimi- nated by applying this equation: $\mathrm{AlbBCG}=5.5+$ AlbBCP [30].

In conclusion, testing for albuminuria should be repeated at least twice to be sure that it is a persistent abnormality. Possible inflammatory causes should be excluded. Semiquantitative regular and albuminuria dipsticks are recommended for screening, but further quantification should be performed using laboratory methods or albumin to creatinine ratio. Moreover, urine albumin concentration should be monitored at regular intervals.

\section{Early kidney injury and other organs}

The assessment of renal abnormalities has a strong role as one of the first steps when evaluating target organ damage in individuals with high cardiovascular risk. Microalbuminuria was found to relate with increased universal vascular sieving of albumin because of transcapillary escape rate, and it may reflect this universal sieving. A possible reason for this behaviour is that albumin leaks through permeant glomeruli exposed to the damaging impact of actually subclinical atherogenesis [31].

Endothelial cell dysfunction is reflected by microalbuminuria especially in hypertensive and diabetic population. The relationship of microalbuminuria with the duration and severity of hypertension, body mass index, age, sex and target organ damage like hypertensive retinopathy, acute coronary syndrome, etc. is emerging. Otherwise, microalbuminuria occurs early in the course of essential hypertension, and ever prehypertension, and this is the overall goal to sample these patients who are at risk for developing chronic disease and its complications.

Inflammatory parameters also play a burning role in target-organ damage in patients with hypertension or high-risk individuals. High-sensitivity C-reactive protein, serum and urinary tumor necrosis factor- $\alpha$ are found to be associated with albuminuria even in prehypertensive subjects, suggesting that inflammation may be a consequential factor for the early vascular damage [32]. In hypertensive patients without diabetes, microalbuminuria or chronic kidney disease, highsensitivity C-reactive protein proved to be a significant predictor of pathologic renal resistive index and decreased renal volume-to-resistive index ratio [33].

Microalbuminuria has a link with target organ damage, especially brain, heart and kidney. Recent studies have shown a close association between kidney and brain in patients with cardiovascular risk. In 3127 stroke-free Framingham off- 
spring patients, investigators identify log-urinary albumin/creatinine ratio to be associated with clinical and subclinical vascular brain injury [34]. Microalbuminuria also is associated with the prevalence of deep or infratentorial brain microbleeds in hypertensive patients without a history of stroke or transient ischemic attack [35]. Other authors show, that ACR is positively related to plaque-initiation and plaque-growth in non-diabetic subjects [36]. Moreover, elevated albuminuria but not GFR is associated with worse cognitive function in young persons in the Prevention of Renal and Vascular End-Stage Disease (PREVEND) study [37]. In elderly patients, albuminuria predicts future modest cognitive decrements, but concurrent albuminuria is unrelated to cognitive functioning [38].

In patients with diabetes, retinopathy is one of the leading causes of blindness that increases the chance of losing the sight. Microalbuminuria is associated with diabetic retinopathy in type II diabetic patients and is a reliable marker of retinopathy [39]. Also it has an impact on predicting the development and progression of retinopathy in these patients [40]. Hypertensive retinal changes of any grade also have accuracy in predicting microalbuminuria, ant this was shown in 870 consecutive hypertensive patients [41].

The association between albuminuria and peripheral artery disease (PAD) has been demonstrated both in general population and in high risk patients with hypertension or diabetes. Nondiabetic adults with albuminuria had a higher prevalence of PAD independently of current renal function after adjustment for multiple cardiovascular risk factors in a large general population study from the National Health and Nutrition Examination Surveys (NHANES) 1999 through 2004 [42].

\section{Conclusions}

CKD is associated with a wide variety of complications and is an independent risk factor for cardiovascular disease. High CKD prevalence within the community at high-risk of ESRD requires a timely screening and proper intervention. A screening strategy for CKD targeting the highest risk groups, those with diabetes or hypertension, family history of diabetes, hypertension, or kidney disease, is likely to be most efficient and cost effective. Complex evaluation of renal function and abnormalities should be done. The most correct GFR estimation should be developed and validated in separate populations, highlighting on MDRD and EPI-CKD equations in patients at-risk. Testing for albuminuria should be repeated and protein quantification should be regularly performed using laboratory methods or albumin to creatinine ratio. The presence of early kidney damage in at-risk groups is associated with target organs damage, thus leading to more detailed examination of these patients.

\section{References}

[1] MacGregor MS. How common is early chronic kidney disease? A background paper prepared for the UK consensus conference on early chronic kidney disease. Nephrol Dial Transplant 2007; 22(suppl 9):ix8-ix18.

[2] Anderson S, Halter JB, Hazzard WR, Himmelfarb J, Horne FM, Kaysen GA et al. Prediction, progression, and outcomes of chronic kidney disease in older adults. J Am Soc Nephrol 2009; 20:1199-1209.

[3] Department of Health. The national service framework for renal services. Part two: chronic kidney disease, acute renal failure and end of life care. COI: London, 2005.

[4] Levey AS, Eckardt KU, Tsukamoto Y, Levin A, Coresh J, Rossert J et al. Definition and classification of chronic kidney disease: a position statement from Kidney Disease: Improving Global Outcomes (KDIGO). Kidney Int 2005; 67:2089-2100.

[5] Toussaint N. Screening for early kidney disease. The CARI guidelines. Published online, 2011, http://www.cari.org.au/DNT\%20workshop\%202011/ 4\%20Screening_Early\%20CKD_DNT.pdf.

[6] Vassalotti JA, Li S, Chen SC, Collins AJ. Screening populations at increased risk of CKD: The Kidney Early Evaluation Program (KEEP) and the public health problem. Am J Kidney Dis 2009; 53:S107-S114.

[7] Ishani A, Grandits GA, Grimm RH, Svendsen KH, Collins AJ, Prineas RJ et al. Association of single measurements of dipstick proteinuria, estimated glomerular filtration rate, and hematocrit with 25-year incidence of end-stage renal disease in the multiple risk factor intervention trial. $\mathrm{J} \mathrm{Am}$ Soc Nephrol 2006; 17:1444-1452.

[8] Kidney Disease Outcomes Quality Initiative. Clinical practice guidelines for chronic kidney disease: evaluation, classification and stratification. Am J Kidney Dis 2002; 39(Suppl 1):S46-S75.

[9] Shafi T, Coresh J. Definition and classification of stages of chronic kidney disease: screening for chronic kidney disease. Cardiorenal syndrome. Springer, 2010.

[10] Mariat C, Maillard N, Phayphet M, Thibaudin L, Laporte $\mathrm{S}$, Alamartine $\mathrm{E}$ et al. Estimated glomerular filtration rate as an end point in kidney transplant trial: where do we stand? Nephrol Dial Transplant 2008; 23:33-38.

[11] Jones GR, Lim EM. The National Kidney Foundation guideline on estimation of the glomerular filtration rate. Clin Biochem Rev 2003; 24:95-98.

[12] Rule A. The CKD-EPI equation for estimating GFR from serum creatinine: real improvement or more of the same? CJASN 2010; 5:951-953.

[13] Nyman H, Dowling T, Hudson J, Wendy L, Joy M, Nolin TD. Use of the Cockcroft-Gault versus the MDRD study equation to dose medications: an opinion of the nephrology practice and research network of the American College of clinical pharmacy. Pharmacotherapy 2011; 31:1130-1144.

[14] Stevens LA, Nolin TD, Richardson MM, Feldman HI, Lewis JB, Rodby $\mathrm{R}$ et al. comparison of drug dosing recommendations based on measured GFR and kidney function estimating equations. AJKD 2009; 54:33-42. 
[15] Vervoort G, Willems H, Wetzels J. Assessment of glomerular filtration rate in healthy subjects and normoalbuminuric diabetic patients: validity of a new (MDRD) prediction equation. Nephrol Dial Transplant 2002; 17:1909-1913.

[16] Delanaye P, Cavalier E, Mariat C, Mailard N, Krzesinski JM. MDRD or CKD-EPI study equations for estimating prevalence of stage $3 \mathrm{CKD}$ in epidemiological studies: which difference? Is this difference relevant? $B M C$ Nephrology 2010; 11:8.

[17] Delanaye P, Cohen E. Formula-based estimates of the GFR: equations variable and uncertain. Nephron Clin Pract 2008; 110:c48-c54.

[18] Levey AS, Stevens LA, Schmid CH, Zhang YL, Castro AF 3rd, Feldman HI et al. A new equation to estimate glomerular filtration rate. Ann Intern Med 2009; 150:604-612.

[19] Matsushita K, Mahmoodi BK, Woodward M, Emberson JR, Jafar TH, Jee SH et al. Comparison of risk prediction using the CKD-EPI equation and the MDRD Study equation for estimated glomerular filtration rate. JAMA 2012; 307:1941-1951.

[20] Kilbride HS, Stevens PE, Eaglestone G, Knight S, Carter JL, Delaney MP et al. Accuracy of the MDRD (Modification of Diet in Renal Disease) study and CKD-EPI (CKD Epidemiology Collaboration) equations for estimation of GFR in the elderly. Am J Kidney Dis 2013; 61:57-66.

[21] Pugliese G, Solini A, Bonora E, Orsi E, Zerbini G, Giorgino F et al. The Chronic Kidney Disease Epidemiology Collaboration (CKD-EPI) equation provides a better definition of cardiovascular burden associated with CKD than the Modification of Diet in Renal Disease (MDRD) study formula in subjects with type 2 diabetes. Atherosclerosis 2011; 218:194-199.

[22] Miller WG, Bruns DE, Hortin GL, Sandberg S, Aakre KM, McQueen MJ et al. Current issues in measurementand reporting of urinary albumin excretion. Clin Chem 2009; 55:24-38.

[23] Miller WG, Bruns DE. Laboratory issues in measuring and reporting urine albumin. Nephrol Dial Transplant 2009; 24:717-718.

[24] Mura-Galelli MJ, Voegel JC, Behr S, Bres EF, Schaaf P. Adsorption/desorption of human serum albumin on hydroxyapatite: a critical analysis of the Langmuir model. Proc Natl Acad Sci USA 1991; 88:5557-5561.

[25] Comper WD, Osicka TM. Detection of urinary albumin. Adv Chronic Kidney Dis 2005; 12:170-176.

[26] Methven S, MacGregor MS, Traynor JP, O'Reilly DS, Deighan CJ. Assessing proteinuria in chronic kidney disease: protein-creatinine ratio versus albumin-creatinine ratio. Nephrol Dial Transplant 2010; 25:2991-2996.

[27] Comper WD, Jerums G, Osicka TM. Differences in urinary albumin detected by four immunoassays and high-performance liquid chromatography. Clin Biochem 2004; 37:105.

[28] Shaikh A, Seegmiller JC, Borland TM, Burns BE, Ladwig PM, Singh RJ et al. Comparison between immunoturbidimetry, size-exclusion chromatography, and LC-MS to quantify urinary albumin. Clin Chem 2008; 54:1504-1510.

[29] McQueen MJ, Gerstein HC, Pogue J, Mann JF, Yusuf S. Reevaluation by high performance liquid chromatography: clinical significance of microalbuminuria in individuals at high risk of cardiovascular disease in the Heart Outcomes Prevention Evaluation (HOPE) study. Am J Kidney Dis 2006; 48:889-896.
[30] Clase CM, St Pierre MW, Churchill DN. Conversion between bromcresol green- and bromcresol purplemeasured albumin in renal disease. Nephrol Dial Transplant 2001; 16:1925-1929.

[31] Pedrinelli R, Penno G, Dell‘Omo G, Bandinelli S, Giorgi D, Di Bello V et al. Microalbuminuria and transcapillary albumin leakage in essential hypertension. Hypertension 1999; 34:491-495.

[32] Chen YH, Chen HS, Tarng DC. More impact of microalbuminuria on retinopathy than moderately reduced GFR among type 2 diabetic patients. Diabetes Care 2012; 35:803-808.

[33] Berni A, Ciani E, Bernetti M, Cecioni I, Berardino S, Poggesi $\mathrm{L}$ et al. Renal resistive index and low-grade inflammation in patients with essential hypertension. J Hum Hypertens 2012; 26:723-730.

[34] Umemura T, Kawamura T, Sakakibara T, Mashita S, Hotta $\mathrm{N}$, Sobue G. Microalbuminuria is independently associated with deep or infratentorial brain microbleeds in hypertensive adults. Am J Hypertens 2012; 25:430-436.

[35] Sierra C, Lopez-Soto A, Coca A. Connecting cerebral white matter lesions and hypertensive target organ damage. J Aging Res 2011; 2011:438978.

[36] Jørgensen L, Jenssen T, Johnsen SH, Mathiesen EB, Heuch $\mathrm{I}$, Joakimsen $\mathrm{O}$ et al. Albuminuria as risk factor for initiation and progression of carotid atherosclerosis in non-diabetic persons: the Tromsø Study. Eur Heart 2007; 28:363-369.

[37] Joosten H, Izaks GJ, Slaets JP, de Jong PE, Visser ST, Bilo HJ et al. Association of cognitive function with albuminuria and eGFR in the general population. Clin J Am Soc Nephrol 2011; 6:1400-1409.

[38] Heringa SM, van den Berg E, Dekker JM, Nijpels G, Kessels $\mathrm{RP}$, Kappelle LJ et al. Albuminuria and cognitive functioning in an older population: The Hoorn Study. Dement Geriatr Cogn Disord 2011; 32:182-187.

[39] Manaviat MR, Afkhami M, Shoja MR. Retinopathy and microalbuminuria in type II diabetic patients. BMC Ophthalmol 2004; 4:9.

[40] Navarro-González JF, Mora C, Muros M, García J, Donate J, Cazaña V. Relationship between inflammation and microalbuminuria in prehypertension. J Hum Hypertens 2013; 27:119-125.

[41] Shantha GP, Kumar AA, Bhaskar E, Sivagnanam K, Srinivasan D, Sundaresan M et al. Hypertensive retinal changes, a screening tool to predict microalbuminuria in hypertensive patients: a cross-sectional study. Nephrol Dial Transplant 2010; 25:1839-1845.

[42] Wu CK, Yang CY, Tsai CT, Chiu FC, Huang YT, Lee JK et al. Association of low glomerular filtration rate and albuminuria with peripheral arterial disease: The National Health and Nutrition Examination Survey, 1999-2004. Atherosclerosis 2010; 209:230-234.

[43] Mensah GA, Croft JB, Giles WH. The heart, kidney, and brain as target organs in hypertension. Cardiol Clin 2002; 20:225-247.

[44] Feldt-Rasmussen B. Microalbuminuria, endothelial dysfunction and cardiovascular risk. Diabetes Metab 2000; 26(Suppl 4):64-66.

[45] Iseki K, Kinjo K, Iseki C, Takishita S. Relationship between predicted creatinine clearance and proteinuria and the risk of developing ESRD in Okinawa, Japan. Am J Kidney Dis 2004; 44:806-814.

[46] Chadban SJ, Briganti EM, Kerr PG, Dunstan DW, Welborn TA, Zimmet PZ et al. Prevalence of kidney damage in Australian adults: The AusDiab kidney study. J Am Soc Nephrol 2003; 14(7 Suppl 2):S131-S138. 
[47] Viktorsdottir O, Palsson R, Andresdottir MB, Aspelund T, Gudnason V, Indridason OS. Prevalence of chronic kidney disease based on estimated glomerular filtration rate and proteinuria in Icelandic adults. Nephrol Dial Transplant 2005; 20:1799-1807.

[48] Garg AX, Kiberd BA, Clark WF, Haynes RB, Clase CM. Albuminuria and renal insufficiency prevalence guides population screening: results from the NHANES III. Kidney Int 2002; 61:2165-2175.

[49] Coresh J, Astor BC, Greene T, Eknovan G, Levey AS. Prevalence of chronic kidney disease and decreased kid- ney function in the adult US population: Third national health and nutrition examination survey. Am J Kid Dis 2003; 41:1-12.

[50] Coresh J, Byrd-Holt D, Astor BC, Briggs JP, Eggers PW, Lacher DA, Hostetter TH. Chronic kidney disease awareness, prevalence, and trends among U.S. adults, 1999 to 2000. J Am Soc Nephrol 2005; 16:180-188.

[51] Hallan SI, Coresh J, Astor BC, Asberg A, Powe NR, Romundstad $\mathrm{S}$ et al. International comparison of the relationship of chronic kidney disease prevalence and ESRD risk. J Am Soc Nephrol 2006; 17:2275-2284. 